\title{
Detecting Human Motion Intention during pHRI Using Artificial Neural Networks Trained by EMG Signals
}

\author{
Doganay Sirintuna ${ }^{1 *}$, Idil Ozdamar ${ }^{1 *}$, Yusuf $\operatorname{Aydin}^{1}$ and Cagatay Basdogan ${ }^{1}$
}

\begin{abstract}
With the recent advances in cobot (collaborative robot) technology, we can now work with a robot side by side in manufacturing environments. The collaboration between human and cobot can be enhanced by detecting the intentions of human to make the production more flexible and effective in future factories. In this regard, interpreting human intention and then adjusting the controller of cobot accordingly to assist human is a core challenge in physical human-robot interaction (pHRI). In this study, we propose a classifier based on Artificial Neural Networks (ANN) that predicts intended direction of human movement by utilizing electromyography (EMG) signals acquired from human arm muscles. We employ this classifier in an admittance control architecture to constrain human arm motion to the intended direction and prevent undesired movements along other directions. The proposed classifier and the control architecture have been validated through a path following task by utilizing a KUKA LBR iiwa 7 R800 cobot. The results of our experimental study with 6 participants show that the proposed architecture provides an effective assistance to human during the execution of task and reduces undesired motion errors, while not sacrificing from the task completion time.
\end{abstract}

\section{INTRODUCTION}

Collaborative robots (cobots) are designed to share a workspace with humans in future factories to complement and/or augment their capabilities [1]. Especially, for the products which can not be manufactured in a fully automated manner, it is envisioned that the physical interaction between humans and cobots (pHRI) will improve the overall efficiency of various manufacturing tasks and processes. It has been argued that, understanding human intention and programming cobot accordingly so that it can adapt itself for the best assistance will make a pHRI system more effective [2]. This will integrate human cognitive skills into the pHRI system, and eventually lead to a more intuitive collaboration and higher task performance.

Human motion intention can be inferred in pHRI by utilizing kinematic and kinetic information such as the velocity of cobot's end-effector and the force applied by human. In addition, there is a direct link between human motion intention and the muscle activation during its contraction which can be characterized by the electromyography (EMG) signals. In this paper, we propose a classifier for human motion direction based on the EMG signals acquired from human arm muscles and an admittance controller architecture

The Scientific and Technological Research Council of Turkey (TUBITAK) supported this work under contract EEEAG-117E645.

* The first two authors contributed equally to this work.

${ }^{1}$ Doganay Sirintuna, Idil Ozdamar, Yusuf Aydin, and Cagatay Basdogan are with College of Engineering, Koc University, 34450, Istanbul, Turkey

\#Corresponding author: cbasdogan aku.edu . tr

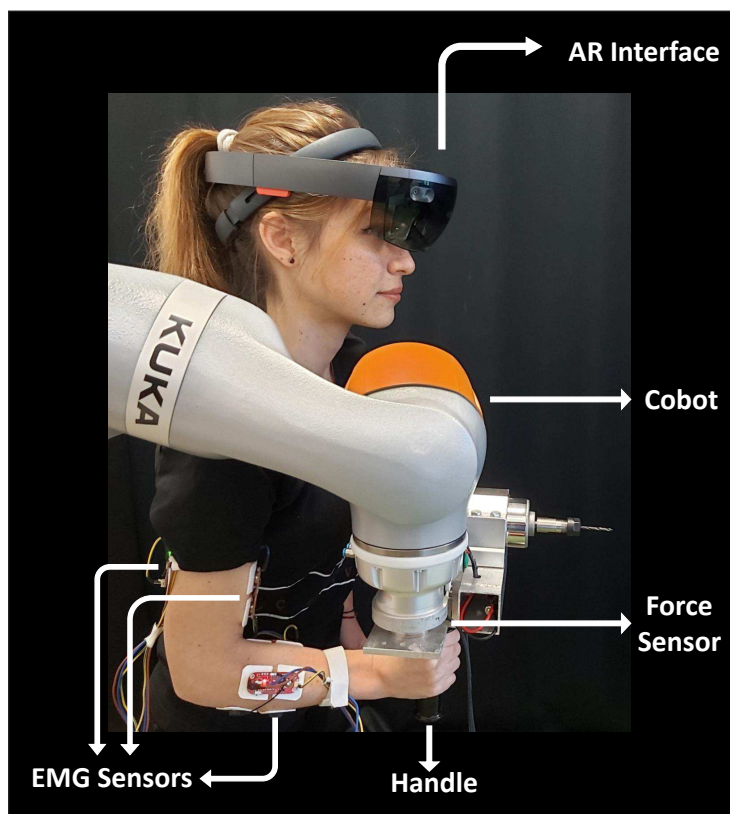

Fig. 1: Our pHRI system.

utilizing this classifier to constrain human arm motion to the intended direction and prevent undesired movements in other directions (see Fig. 1).

Such an architecture, for example, could be beneficial when a human operator in a factory aims to drill a series of holes at the corners of a rectangular workpiece where a cobot can constrain the movements of the operator to help with the task [3]. In such a setting, the operator grabs the power drill attached to the end-effector of the cobot and brings it closer to the surface of the workpiece. This requires a free space movement with no constraints in any direction. Then, the contact occurs and drilling of a hole at one corner begins. At this stage, the cobot should constrain the movement to the surface normal for precise drilling. To drill another hole at a nearby corner, the cobot may allow horizontal/vertical movement in the direction from the first corner to the second corner only, so that the operator moves the drill easily along that direction until the second one is reached. Then, the cobot allows the movement along the surface normal only again, so that the second hole can be drilled at that corner. This procedure can be repeated until all four holes are drilled at the corners. As can be seen from this example, the cobot needs to constrain the movements of the operator to the horizontal and vertical directions on the surface of workpiece during the movements from one corner to another and also 


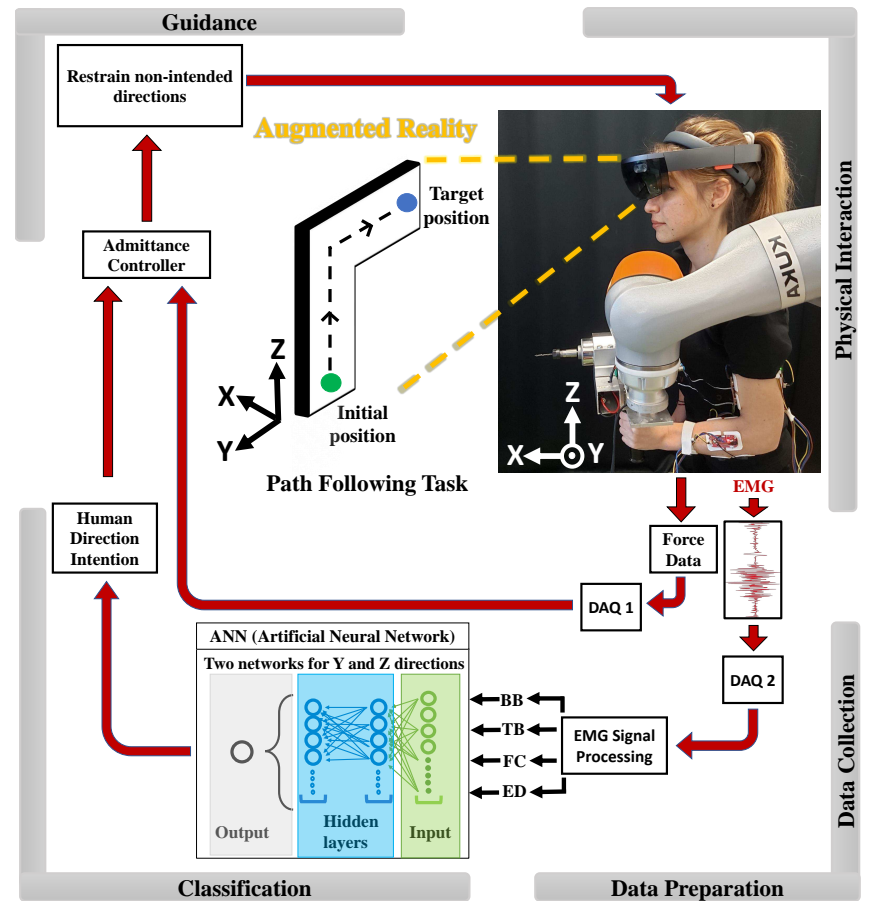

Fig. 2: Our closed-loop controller architecture that cooperates with the proposed motion direction classifier for providing a guidance to human operator during the collaborative path following task. The path to be followed is displayed through an augmented reality interface.

to the direction perpendicular to the surface while opening a hole.

To this end, our approach can estimate horizontal and vertical arm movements of an operator, and then we can adjust the admittance controller of a cobot accordingly to allow movements along those directions only, so that a collaborative task can be performed efficiently and precisely. In order to investigate the effectiveness of the proposed approach, we conduct a pHRI experiment in a laboratory environment. This experiment can be considered as an abstraction of the actual drilling scenario given above. In our experiment, the participants hold a handle rigidly attached to the end-effector of a KUKA LBR iiwa 7 R800 cobot to follow a given path (see Fig. 2). The path involves horizontal and vertical movements on a virtual plane $(Y-Z$ plane in Fig. 2). Both the path and the instantaneous position of the cobot's end-effector are visually displayed through an AR interface (HoloLens, Microsoft Inc.) during the experiment. With this experiment, we show that intended movements of a human operator can be estimated in real-time by utilizing artificial neural networks (ANN) trained by EMG signals only. This estimation can be then used by our cobot to guide the operator to perform the path following task with less error, compared to no guidance case.

\section{A. Related Work}

In the literature, interaction (impedance/admittance) controllers are typically utilized to regulate the physical interaction between human and cobot. During this interaction, if the intention of human is detected, the controller parameters can be adjusted on the fly to increase the overall performance of the pHRI system.

In order to detect the intention of human, kinematic information such as velocity can be used. For instance, Ikeura et al. [4] employed the velocity of manipulated object to change the damping value of an admittance controller. Similarly, Kang et al. [5] utilized object's velocity to presume whether the operator intends to accelerate or decelerate the manipulated object, and then altered the damping parameter of an admittance controller accordingly.

In pHRI, kinetic information such as force is also used for anticipating the intention of human. Li et al. [6], [7] estimated the intention based on the force exerted by human, and then altered the controller parameters accordingly. Wakita et al. [8] anticipated the intended movement direction based on the interaction force between human and robot and designed an adaptive admittance controller to eliminate undesired motions during assisted-walking. Instead of using force alone, combination of object's velocity and force derivative were also utilized to foresee whether human intends to accelerate or decelerate the manipulated object [9], [10].

The main drawback of utilizing kinetics (such as force) and kinematics (such as velocity) based information in intent detection is that they are acquired right after human starts realizing her/his intended motion. Hence, there exists an inevitable delay in intent detection.

As an alternative, EMG-based information provides an opportunity to detect human intention even before the movement starts. In skeletal muscles (those under the voluntary control of the somatic nervous system), EMG activity precedes the motion of actuated limb. The delay in limb motion is due to the time lag between the onsets of muscle electrical activation and force production, which is in the range of 50-100 ms [11], [12]. In other words, EMG signals can provide information about the intended human motion before its initiation. This early detection possibility may improve the response time of cobot in pHRI and lead to more natural cooperation [13].

Besides the fact that EMG activity precedes the limb movement, it also provides a rich neural information about the intended human motion [14]. These make EMG a valuable signal for pHRI, especially in the areas of exoskeletons and rehabilitation robotics. The research studies in those areas typically aim to design an actuator system that reduces human muscular effort by adjusting its controller based on EMG signals. For instance, $\mathrm{Li}$ et al. [15] adjusted the torque control signals of an exoskeleton by estimating human motion intent based on EMG signals. Karavas et al. [16] utilized an EMG-driven musculoskeletal model to interpret human movement intention in order to adjust the assistive torques of a knee exoskeleton. Tang et al. [17] predicted the elbow angle of human arm in real-time based on EMG signals which was then utilized by the controller of an exoskeleton to augment human arm performance.

In comparison to the studies above, the number of pHRI studies on object co-manipulation that utilize EMG-based 
information for detecting human intention is limited. DelPreto and Rus [18] designed a control framework to provide effective assistance based on EMG information of the upper arm in a collaborative lifting task. Grafakos et al. [19] proposed a variable admittance controller to establish an effortless collaboration between human and cobot based on muscle activation of the operator. $\mathrm{Wu}$ et al. [20] designed a variable impedance controller based on EMG signals for teaching a cobot how to perform collaborative tasks with human.

\section{B. Contributions}

In our approach, we continuously acquire the muscle activation levels (i.e. EMG signals) of human arm during the execution of a pHRI task, imitating collaborative drilling, to detect the direction of human arm movement via ANN. This information is then used to constrain the arm movement to the intended direction only via an admittance controller to help with the execution of task. To our knowledge, no prior studies have proposed a classifier to provide such guidance to human operator during a collaborative pHRI task. As mentioned earlier, EMG signal precedes force and position signals and helps to identify the motion intent even before the movement starts. This creates an opportunity for us to adapt our admittance controller on-the-fly to comply with human intention swiftly in our pHRI task.

The rest of this article is organized as follows: Section II presents our approach. Section III explains our pHRI experiment in detail. It covers our experimental setup and scenario, EMG data acquisition and signal processing, and the artificial neural network structure for the classification. We present the experimental procedure for collecting data to train our classifier and employ it with the proposed control architecture in real-time during a path following task in the same section. Section IV reports the prediction accuracies of our classifier during offline training and online evaluation. Then, it presents the performance results of the proposed control architecture with and without guidance for the path following task. Lastly, in Section V, we discuss the results of the path following task, and conclude the study with possible future research directions.

\section{APPROACH}

In a typical pHRI setting, an operator and a cobot physically interact with each other and/or their environment to accomplish a task. To regulate the interaction between human, robot, and environment, an admittance control architecture is widely used. In this architecture, the motion of cobot is determined based on the forces applied by human. However, even if the human, for example, aims to move on a straight line, the cobot is sensitive to small forces exerted by the human and positional deviations from the desired path may occur. To eliminate these deviations and create more precise motion, human movement intention can be detected to adapt an interaction controller. In particular, if the intended movement direction is detected accurately, then the cobot may be constrained to move along that direction only.
For the implementation of the path following scenario discussed above, we integrate the admittance control architecture suggested in [21]-[23] with the proposed direction classifier. So, the controller enables movements along the intended directions of human arm only that are estimated using ANN, trained by EMG signals alone (see Fig. 2). Note that the parameters of this controller remain fixed whereas the movement direction is altered on the fly during the pHRI task. In our implementation, the admittance controller along each Cartesian direction $i$ is given by:

$$
\frac{v_{\mathrm{ref}}^{i}}{F_{h}^{i}}=\frac{1}{m s+b}
$$

where $i \in[X, Y, Z], s$ is the Laplace variable, $m$ and $b$ are the admittance mass and damping parameters, respectively, $F_{h}^{i}$, and $v_{\text {ref }}^{i}$ are the force applied by operator and the reference velocity command to cobot along the direction $i$, respectively. Once the intended movement direction is detected, the forces applied by the operator in other directions are set to zero. For example, if the operator intends to move in $Y$-direction only, the force tangent to the movement direction $(Z)$ and normal to the movement plane $(X)$ are simply set to zero (see Fig. 2).

In order to estimate intended movement direction, muscle activation levels of human arm can provide rich information. To this end, we place four EMG sensors on various muscles of the human arm and monitor the acquired EMG data. At each time step $t$, the collected measurements from these sensors create a feature vector given by $x_{t}$. Because a single data point cannot provide sufficient information to estimate motion intent [24], time window was employed. So, the classification problem is formulated as follows: does human operator intend to move along a certain direction given a window of data points of size $w$ containing $\left[x_{t-w+1}\right.$, $\left.x_{t-w+2}, \ldots ., x_{t}\right]$ ? In our implementation, we train Artificial Neural Networks (ANN) to decide whether there is an intended movement along the direction(s) of $Y$ and/or $Z$. Based on this, we show that it is possible to detect intended movement directions offline by utilizing our approach. After achieving satisfactory offline accuracy, we utilize the trained model to capture the intended movement directions of 6 participants in real-time during the path following task.

\section{EXPERIMENT}

\section{A. Experimental Setup}

Our experimental setup consists of 4 surface EMG (sEMG) sensors (MyoWare Muscle Sensor AT-04-001, Advancer Technologies Inc.), a force sensor (Mini40, ATI Inc.), and a custom-made handle attached to the end-effector of a LBR iiwa 7 R800 robot (KUKA Inc.) as shown in Fig. 1. The raw EMG signals were collected using DAQ card (USB-6251 NI Inc.) at $2 \mathrm{kHz}$. A separate DAQ card (PCI-6225, NI Inc.) was used to acquire data from the force sensor at $10 \mathrm{kHz}$. The closed-loop control architecture given in Fig. 2 was updated at $250 \mathrm{~Hz}$. In order to achieve this high update rate for the control-loop, and ensure stable and reliable communication, 

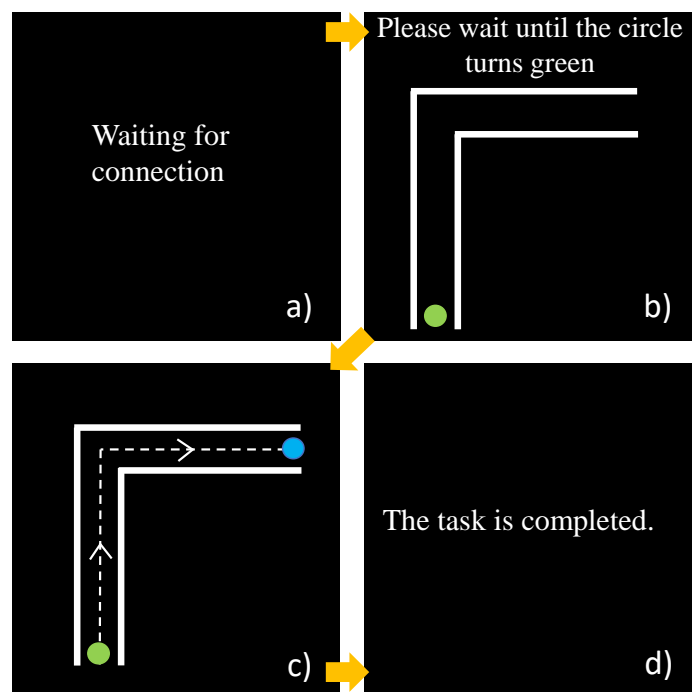

Fig. 3: Visual information displayed to the participants during the task execution through the AR interface. Green and blue circles in (c) indicate start and target positions, respectively, and dashed-lines with arrows represent the desired trajectory.

the cobot's Fast Robot Interface (FRI) library was used. We implemented an admittance controller by using the "Joint Position Controller" function of the library in C/C++ environment [25] with the help of the forward and inverse kinematics reported in [26].

\section{B. Experimental Scenario}

During the experiment, visual feedback was provided to the participants through the AR interface about the steps of the task (Fig. 3). First the visual scene informed the participants about the connection between the AR interface and the cobot (Fig. 3a). Once the connection was established, a path to be followed by the participants and its starting point (a red circle) were displayed. For each path, the cobot traveled to the starting point of the path automatically. The color of the starting point remained red until the cobot reached it and stopped there. The participants were informed not to hold the handle until the circle turns green (Fig. 3b). Once the circle color became green, the participants were able to track the given path along the indicated directions (see the dashed lines in Fig. 3c). In our experiment, the cobot was constrained to move in $Y-Z$ plane only (see Fig. 2). To create different paths which include two consecutive movements in $Y$ and $Z$ directions with different combinations, we split a square path into 8 sub-paths as shown in Fig. 4. Each sub-path consisted of 2 segments in $Y$ and $Z$ directions. The length and width of each segment were $25 \mathrm{~cm}$ and $2 \mathrm{~cm}$, respectively.

\section{Placement of EMG Sensors and Signal Processing}

Working with EMG sensors requires additional care as the subsequent processes and the accuracy of continuous motion parameter estimation primarily depend on the quality and correctness of the acquired signal. Hence, the sections below explain in detail; the procedure that we followed for EMG placement on the muscles, EMG signal recording and

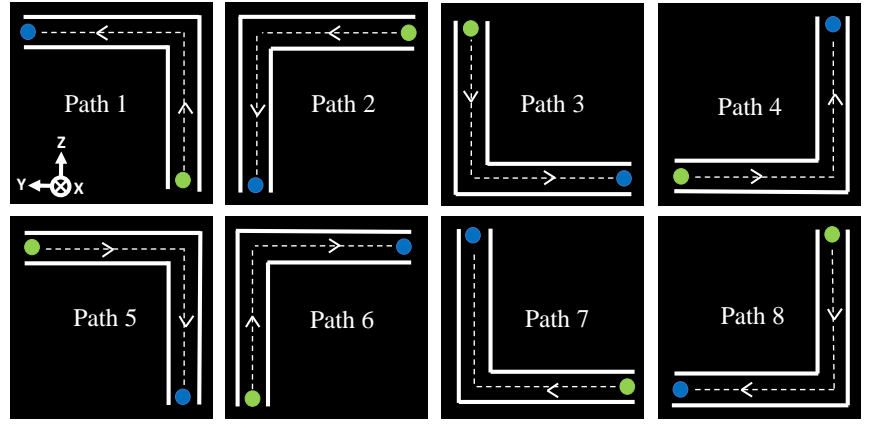

Fig. 4: The paths to be followed in the experiments. Green and blue circles indicate start and target positions, respectively, and dashed lines with arrows represent the desired motion trajectories.

processing, and the normalization that enables comparisons across different subjects.

1) Sensor Positions and Placement: Biceps Brachii (BB) short head, Triceps Brachii (TB) long head, Flexor Carpi (FC) and Extensor Digitorum (ED) were selected as the muscle groups for this study. We used only the participant's arm muscles that were related to the execution of 4 movements in our task $(+Z$ : up, $-Z$ : down, $+Y$ : left, $-Y$ : right). Four sEMG sensors and differential pairs of adhesive electrodes were placed on those muscles of participants' right arm. A good electrode-skin contact is required to obtain better EMG recordings in terms of fewer and smaller artefacts (electrical interference), less risk of imbalance between electrodes and better signal to noise ratio (SNR). For these reasons, we first removed the hair on the arm skin where the electrodes were placed and then cleansed that area with alcohol in accordance with the SENIAM recommendations for skin preparation [27]. Sensors were placed on the longitudinal midline of each muscle parallel to the direction of fibers for gathering high quality signals and to obtain repeatable measurements [28]. The distance between the sEMG sensors was always more than $2 \mathrm{~cm}$ to prevent muscle crosstalk [29].

2) EMG Signal Processing and Normalization: First, raw EMG data was acquired at $2 \mathrm{kHz}$ in order to avoid any loss of muscular information [30]. Second, the collected

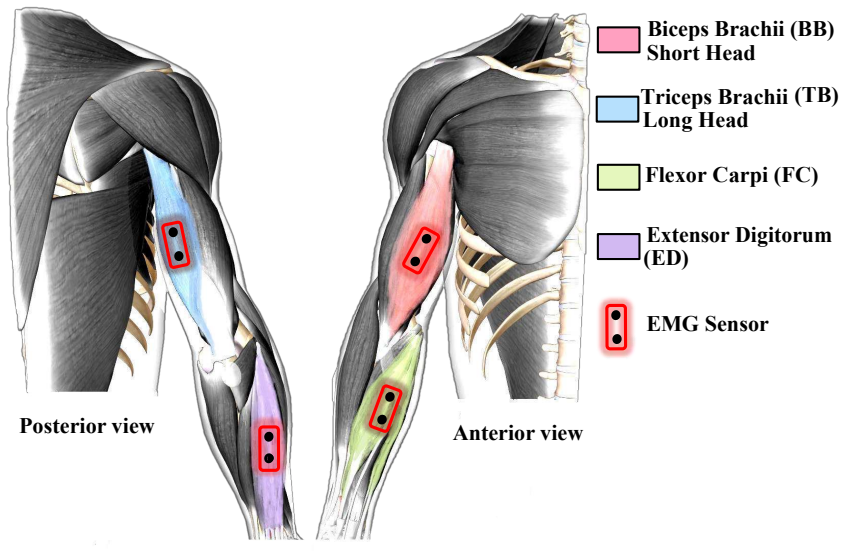

Fig. 5: Placements of electrodes in our study for acquisition of EMG data. 
EMG signals were filtered with a band-pass Butterworth filter having lower and upper cutoff frequencies of $5 \mathrm{~Hz}$ and $500 \mathrm{~Hz}$, respectively. The lower cutoff of the filter removed the offset and reduced the movement artifacts of the EMG signal [31] since low-frequency content distorts the true nature of surface EMG signal [32]. The upper cutoff helped to get rid of high frequency noise while capturing muscles' full informative frequency spectrum [28]. Third, we applied full-wave rectification by taking absolute value of the filtered EMG signals. Compared to half-wave rectification, which eliminates all the negative parts of the signal, fullwave rectification was preferred since it conserves all of the signal energy [14]. Fourth, a low-pass Butterworh filter having a cutoff frequency of $5 \mathrm{~Hz}$ was applied to the rectified signal for extraction of the signal envelope [18]. Lastly, the resulting signals were normalized by using Peak Dynamic Method (PDM) to be able to compare EMG activity in the same muscle on different days or in different individuals or between muscles [33], [34]. PDM expresses EMG data from a muscle as a ratio of the peak value acquired from the same muscle during the task under investigation and produces good reliability between sessions and between subjects [35]-[37], and reduces inter-subject variability [35]. In addition, normalization rescales the raw EMG amplitudes from millivolts into a percentage of this reference value [38].

\section{Artificial Neural Networks (ANN)}

The activation of each muscle contributes non-linearly to a movement activity [39], [40]. To overcome such drawback, ANN are commonly utilized for movement classification since they can detect associations or regularities among the data which have complex relationships [41].

In this study, we considered arm movements along $Y$ and $Z$ directions on a virtual plane (see Fig. 2). For this reason, we trained two separate networks. Network 1 was responsible for detecting the intended movements in $Y$ direction, whereas Network 2 was utilized for $Z$ direction. Output of each neural network was whether the movement existed in the corresponding direction or not. If the detected movement was along $Y(Z)$ axis only, then the controller constrained the movements to $Y(Z)$ and $X$ axis. On the other hand, if both networks detected movements, then the controller allowed movements on the entire $Y-Z$ plane and constrained the movements to $X$ axis only.

As mentioned in Section II, we used a running window of data points to train our networks. In this study, we selected this window size as 25 . Hence, our ANN have 100 input nodes each, since the data was collected from 4 EMG sensors ( 4 x 25). Each network structure has 2 hidden layers with 50 nodes each utilizing a Rectified Linear Unit activation function (ReLU). We used Adam's update rule with a learning rate of 0.001 . The output layer of the neural network has a single unit, and utilizes a sigmoid activation function.

\section{E. Offline Training and Testing}

The first part of the experiment was designed to collect sufficient and diverse data to train and test our ANN-based classifier offline.

1) Procedure: This part of the experiment was conducted with two participants ( 1 female and 1 male), who were 25 years old. For each participant, there were a total of 96 trials ( 8 paths $\times 4$ repetitions per path $\times 3$ sessions) displayed in random order. The sessions for each participant were executed in 3 consecutive days. Participants were asked to follow the path as straight as possible and focus on precision rather than elapsed time. The admittance controller constrained the movements of participants to $X$ axis but not in $Y-Z$ plane. They were asked to stop at the corners for 2 seconds so that the movements along $Y$ (left-right) and $Z$ (up-down) axes can be distinguished easily during data labeling.

In total, there were 24 trials ( 4 repetitions $x 3$ sessions $\mathrm{x} 2$ participants) for each path and 18 (3 repetitions $\mathrm{x} 3$ sessions $\mathrm{x} 2$ participants) of them were considered in training and the remaining were used in testing. In other words, $75 \%$ of the data was used for the training and $25 \%$ for the testing.

\section{F. Online Evaluation}

In the second part of the experiment, we aimed to evaluate the real-time performance of our proposed architecture using the classifier trained in the first part. For this purpose, we compared the unintentional positional deviations from the desired paths under the admittance controller with guidance (based on the detected direction) to that of without guidance. Furthermore, the participants whose data were used for the training of the classifier in the first part did not attend this part of the experiment, in order to check if the trained model is robust and insensitive to the data of new participants.

1) Procedure: This part of the experiment was conducted with 6 participants (all right-handed, 3 females, average age $=23 \pm 2.3$ ). Each participant performed a total of 32 trials ( 4 different paths $\times 2$ controllers $\times 4$ repetitions). To avoid a long and tiring experiment, we conducted the experiment with 4 paths only (Paths 1, 2, 3, and 4 in Fig. 4), which included all types of straight movements in $Y-Z$ plane (right to left, left to right, up to down and down to up). The paths and the controllers were presented to the participants in a randomized order. In the first eight trials, all four paths and two controllers (with and without guidance) were presented to the participants once to familiarize them with the setup and the task, and no data was collected during those trials. As in the first part, the participants were asked to follow the path as straight as possible and focus on precision rather than elapsed time.

\section{RESULTS}

\section{A. Offline Training and Testing}

The classifiers of $Y$ and $Z$ directions reached nearly the same accuracies of $96.6 \%$ and $96.8 \%$ respectively during the testing data. Fig. 6 shows the confusion matrices of the classifiers. This high recognition accuracy encouraged us that 


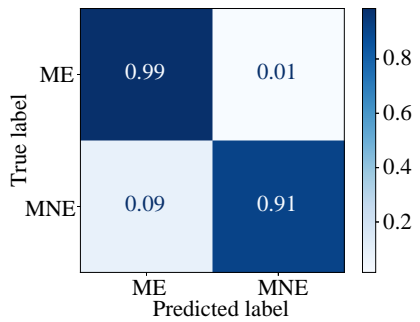

(a)

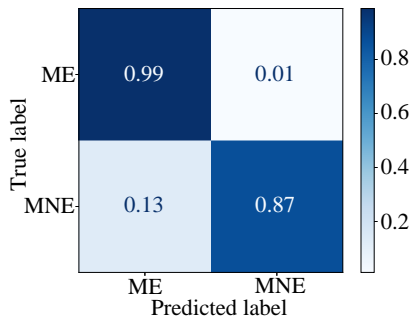

(b)
Fig. 6: Confusion matrices for the offline training part of the experiment along (a) $Y$ direction and (b) $Z$ direction (ME: Movement exists, MNE: Movement does not exist.)

EMG signal could be used alone to detect intended direction of human movement during a real-time application.

\section{B. Online Evaluation}

The real-time detection accuracies of the classifiers along $\mathrm{Y}$ and $\mathrm{Z}$ directions were $85.6 \%$ and $89.3 \%$, respectively. The normalized confusion matrices in Fig. 7 show that the accuracies of the classifiers in detecting the existence and nonexistence of the motion in each direction are similar.

To evaluate the task performance under two different controllers, we calculated the accumulated deviations from linearity (denoted by $e$ ), by summing up the absolute value of movement velocity that is orthogonal to the current path, as follows:

$$
e=\sum_{t_{b}}^{t_{e}}\left|v_{\perp}(t)\right|
$$

where $\perp$ represents the direction orthogonal to the current path, and $t_{b}$ and $t_{e}$ are the beginning and ending times of a trial, respectively. Furthermore, we investigated how the task completion time changed with the controller type, though, no instruction was given to the participants about task execution speed.

The means and standard errors of the means for deviation from linearity $\left(e^{\text {ave }}\right)$ and task completion time $\left(t^{\text {ave }}\right)$ are shown in Fig. 8a and 8b, respectively, for each controller type. A one-way ANOVA with repeated measures was performed to test the effect of controller type as the main factor

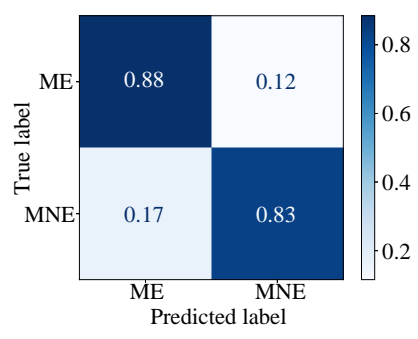

(a)

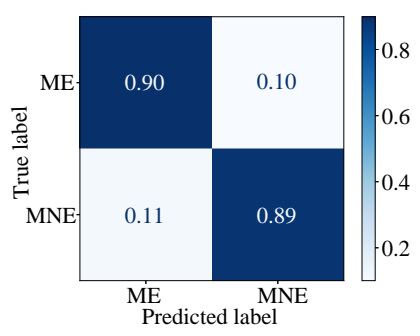

(b)
Fig. 7: Confusion matrices for the online evaluation part of the experiment along (a) $Y$ direction and (b) $Z$ direction (ME: Movement exists, MNE: Movement does not exist.)

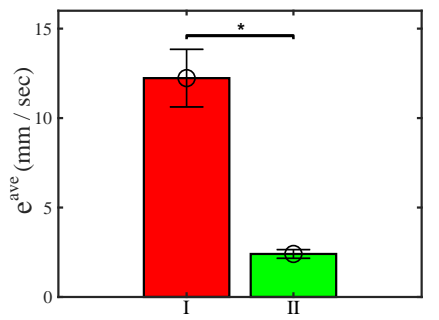

(a)

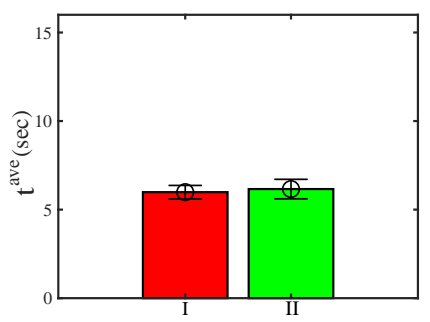

(b)
Fig. 8: The means (average of all participants) and standard errors of means for the metrics of a) deviation from linearity and b) task completion time. (I: Without direction classifier, II: With direction classifier. Horizontal bar with $*$ on top indicates statistical significance between the results of the two corresponding conditions.)

on $e^{\text {ave }}$ and $t^{\text {ave }}$. A significance level of $p<0.005$ was used to test the null hypothesis. The results showed that, $e^{\text {ave }}$ for the controller with guidance utilizing the classifier $\left(F_{1,5}=\right.$ $42.88, \mathrm{p}=0.001$ ) was significantly lower than that of the controller without guidance (see Fig. 8a). However, there was no significant effect of controller type on task completion time $\left(F_{1,5}=0.14, \mathrm{p}=0.73\right)$.

\section{DISCUSSION AND CONCLUSIONS}

A good sensory communication between human operator and cobot is vital for more effective pHRI. Real-time detection of human movement intention can be highly beneficial for achieving this aim. In this study, we focused on detection of motion direction intention and developed a classifier to predict it in real-time by utilizing ANN, which take EMG data alone as the input for training. We showed that our direction classifier has high detection accuracy.

The effectiveness of the proposed classifier was evaluated for a path following task. As discussed in Section II, human operator easily deviate from a desired path during the execution of a collaborative manipulation task executed with a cobot. To demonstrate these deviations, we present the position data of one participant during the tracking of a path under the admittance controller with no guidance (Fig. 9). In this task, the participant aims to follow a straight line along $Z$ axis first, and then changes his direction to move along $Y$ axis (see path 1 in Fig. 4). As seen in close-ups, even though the intent of the participant is to move on $Y(Z)$ axis, there are unintentional positional deviations in $Z(Y)$ axis. To prevent those deviations, we constrain the movement to the intended direction using the admittance controller with guidance as discussed in Section II.

We showed that the admittance controller with guidance (allowing only the movements along the directions predicted by the proposed classifier while preventing the movements in other directions) outperformed the admittance controller with no guidance. The admittance controller with direction classifier resulted in a significant decrease in undesirable deviations from the desired path (Fig. 8a). Moreover, we would like to highlight that our direction classifier was robust to the data of new participants. Although all the participants 


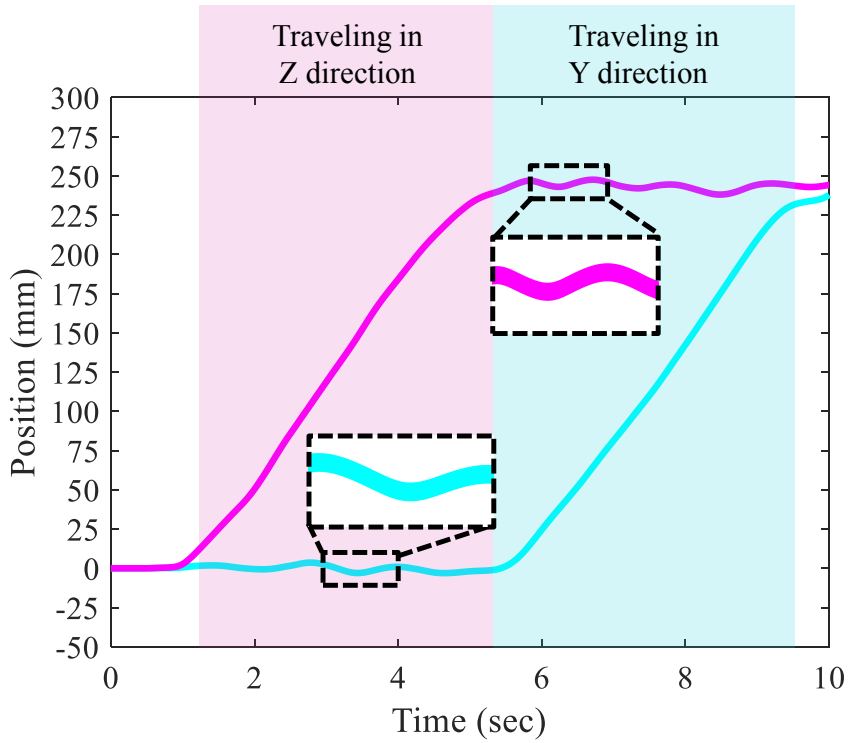

Fig. 9: Position data of a participant as it follows the path 1 given in Fig. 4 under the admittance controller with no guidance. The participant moves in $+Z$ direction (light pink) first, and then moves in $+Y$ direction (light blue) later. Close-ups (in dashed boxes) show the positional deviations in $Z(Y)$ while the participant aims to travel only along $Z(Y)$ direction. The targeted travel directions are illustrated using the shaded regions.

in the online evaluation part of the experiment were new and none of their data was used for the training of the classifier, it still successfully detected the direction intention of each participant.

In the future, we plan to execute a drilling task on a real workpiece to demonstrate the practical applicability of our approach. It will involve movements in the direction of drilling axis ( $X$ axis) in addition to the movements on the surface of workpiece $(Y-Z$ plane) as in this study. In order to accomplish such a task, we need to detect motion intention along the drilling direction by training a separate network for $\mathrm{X}$ axis. For this purpose, acquiring EMG data from different muscle groups such as Anterior Deltoid might be required. Furthermore, we plan to combine our proposed architecture with a variable admittance controller as implemented in [3]. Instead of using an admittance controller with fixed parameters as in this study, the controller parameters will be adjusted on-the-fly according to the needs of human operator, which will be estimated based on kinetic/kinematic information. For instance, admittance damping could be increased (decreased) to allow precise positioning (effortless collaboration) during the task, as needed.

\section{ACKNOWLEDGMENT}

The Scientific and Technological Research Council of Turkey (TUBITAK) supported this work under contract EEEAG-117E645. Moreover, the authors thank to Dr. O. Caldiran for the fruitful technical discussions during this study and B. Oznalbant, and B. Tosun for the initial support.

\section{REFERENCES}

[1] A. Ajoudani, A. M. Zanchettin, S. Ivaldi, A. Albu-Schäffer, K. Kosuge, and O. Khatib, "Progress and prospects of the human-robot collaboration," Autonomous Robots, vol. 42, pp. 957-975, Oct. 2018.

[2] D. P. Losey, C. G. McDonald, E. Battaglia, and M. K. O'Malley, "A Review of Intent Detection, Arbitration, and Communication Aspects of Shared Control for Physical Human-Robot Interaction," Applied Mechanics Reviews, vol. 70, no. 1, Jan. 2018.

[3] D. Sirintuna, Y. Aydin, O. Caldiran, O. Tokatli, V. Patoglu, and C. Basdogan, "A variable-fractional order admittance controller for phri," Proceedings of IEEE International Conference on Robotics and Automation (ICRA), pp. $10162-10168$, May 2020.

[4] R. Ikeura, T. Moriguchi, and K. Mizutani, "Optimal variable impedance control for a robot and its application to lifting an object with a human," in IEEE International Workshop on Robot and Human Interactive Communication, pp. 500-505, Sep. 2002.

[5] G. Kang, H. S. Oh, J. K. Seo, U. Kim, and H. R. Choi, "Variable admittance control of robot manipulators based on human intention," IEEE/ASME Transactions on Mechatronics, vol. 24, no. 3, pp. 10231032, June 2019.

[6] H. Li, I. Paranawithana, L. Yang, T. S. K. Lim, S. Foong, F. C. Ng, and U. Tan, "Stable and compliant motion of physical human-robot interaction coupled with a moving environment using variable admittance and adaptive control," IEEE Robotics and Automation Letters, vol. 3, no. 3, pp. 2493-2500, July 2018.

[7] H. Li, T. Nuradha, S. A. Xavier, and U. Tan, "Towards a compliant and accurate cooperative micromanipulator using variable admittance control," in International Conference on Advanced Robotics and Mechatronics (ICARM), July 2018, pp. 230-235.

[8] K. Wakita, J. Huang, P. Di, K. Sekiyama, and T. Fukuda, "Humanwalking-intention-based motion control of an omnidirectional-type cane robot," IEEE/ASME Transactions on Mechatronics, vol. 18, no. 1, pp. 285-296, Feb. 2013.

[9] V. Duchaine and C. M. Gosselin, "General model of human-robot cooperation using a novel velocity based variable impedance control," in Second Joint EuroHaptics Conference and Symposium on Haptic Interfaces for Virtual Environment and Teleoperator Systems (WHC'07), pp. 446-451, March 2007.

[10] V. Duchaine, B. Mayer St-Onge, D. Gao, and C. Gosselin, "Stable and intuitive control of an intelligent assist device," IEEE Transactions on Haptics, vol. 5, no. 2, pp. 148-159, April 2012.

[11] P. Artemiadis, "Emg-based robot control interfaces: Past, present and future," Advances in Robotics \& Automation, vol. 1, Jan. 2012.

[12] P. Cavanagh and P. Komi, "Electromechanical delay in human skeletal muscle under concentric and eccentric contractions," European Journal of Applied Physiology and Occupational Physiology, vol. 42, no. 3, pp. 159-163, Nov. 1979.

[13] T. Tamei, S. Ishii, and T. Shibata, "Virtual force/tactile sensors for interactive machines using the user's biological signals," Advanced Robotics, vol. 22, no. 8, pp. 893-911, Aug. 2008.

[14] L. Bi, F. A. Genetu, and C. Guan, "A review on emg-based motor intention prediction of continuous human upper limb motion for humanrobot collaboration," Biomed. Signal Proc. and Control, vol. 51, pp. 113-127, May 2019.

[15] Z. Li, B. Wang, F. Sun, C. Yang, Q. Xie, and W. Zhang, "sEMGbased joint force controls for an upper-limb power-assist exoskeleton robot," IEEE Journal of Biomedical and Health Informatics, vol. 18 , pp. 1043-1050, May 2014.

[16] N. Karavas, A. Ajoudani, N. Tsagarakis, J. Saglia, A. Bicchi, and D. Caldwell, "Tele-impedance based assistive control for a compliant knee exoskeleton," Robotics and Autonomous Systems, vol. 73, pp. 78 - 90, Nov. 2015.

[17] Z. Tang, K. Zhang, S. Sun, Z. Gao, L. Zhang, and Z. Yang, "An upperlimb power-assist exoskeleton using proportional myoelectric control," Sensors, vol. 14, pp. 6677-6694, Apr. 2014.

[18] J. DelPreto and D. Rus, "Sharing the load: Human-robot team lifting using muscle activity," International Conference on Robotics and Automation (ICRA), pp. 7906-7912, May 2019.

[19] S. Grafakos, F. Dimeas, and N. Aspragathos, "Variable admittance control in phri using emg-based arm muscles co-activation," in IEEE International Conference on Systems, Man, and Cybernetics (SMC), pp. 1900-1905, Oct 2016.

[20] R. Wu, H. Zhang, T. Peng, L. Fu, and J. Zhao, "Variable impedance interaction and demonstration interface design based on measurement 
of arm muscle co-activation for demonstration learning," Biomedical Signal Processing and Control, vol. 51, pp. 8 - 18, May 2019.

[21] Y. Aydin, O. Tokatli, V. Patoglu, and C. Basdogan, "Fractional order admittance control for physical human-robot interaction," in IEEE World Haptics Conference (WHC), June 2017, pp. 257-262.

[22] Y. Aydin, O. Tokatli, V. Patoglu, and C. Basdogan, "Stable physical human-robot interaction using fractional order admittance control," IEEE Transactions on Haptics, vol. 11, no. 3, pp. 464-475, July 2018.

[23] Y. Aydin, O. Tokatli, V. Patoglu, and C. Basdogan, "A computational multi-criteria optimization approach to controller design for physical human-robot interaction," IEEE Transactions on Robotics, to appear, available: arXiv:2006.11218.

[24] M. A. Oskoei and H. Hu, "Myoelectric control systems-a survey," Biomedical Signal Processing and Control, vol. 2, no. 4, pp. 275 294, Oct. 2007.

[25] Y. Aydin, D. Sirintuna, and C. Basdogan, "Towards collaborative drilling with a cobot using admittance controller," Transactions of the Institute of Measurement and Control, to appear.

[26] C. Faria, F. Ferreira, W. Erlhagen, S. Monteiro, and E. Bicho, "Position-based kinematics for 7-dof serial manipulators with global configuration control, joint limit and singularity avoidance," Mechanism and Machine Theory, vol. 121, pp. 317-334, March 2018.

[27] H. J. Hermens, B. Freriks, C. Disselhorst-Klug, and G. Rau, "Development of recommendations for semg sensors and sensor placement procedures," Journal of Electromyography and Kinesiology, vol. 10, no. 5, pp. 361 - 374, Oct. 2000.

[28] C. J. D. Luca, "Surface electromyography: Detection and recording," DelSys Incorporated, vol. 10, no. 2, 2002.

[29] Y. Mon and A. Al-Jumaily, "Estimation of upper limb joint angle using surface emg signal," International Journal of Advanced Robotic Systems, vol. 10, p. 1, Oct. 2013.

[30] N. Dimitrova, T. Arabadzhiev, J.-Y. Hogrel, and G. Dimitrov, "Fatigue analysis of interference emg signals obtained from biceps brachii during isometric voluntary contraction at various force levels," Journal of Electromyography and Kinesiology, vol. 19, no. 2, pp. 252 - 258, Apr. 2009.

[31] C. J. D. Luca, L. D. Gilmore, M. Kuznetsov, and S. H. Roy, "Filtering the surface emg signal: Movement artifact and baseline noise contamination," Journal of Biomechanics, vol. 43, no. 8, pp. 1573 1579, May 2010.

[32] S. H. Brown, R. L. Brookham, and C. R. Dickerson, "High-pass filtering surface emg in an attempt to better represent the signals detected at the intramuscular level," Muscle \& Nerve, vol. 41, no. 2, pp. 234-239, Feb. 2010.

[33] C. J. D. Luca, "The use of surface electromyography in biomechanics," Journal of Applied Biomechanics, vol. 13, no. 2, pp. 135-163, Jan. 1997.

[34] G. L. Soderberg and L. M. Knutson, "A Guide for Use and Interpretation of Kinesiologic Electromyographic Data," Physical Therapy, vol. 80, no. 5, pp. 485-498, May 2000.

[35] J. F. Yang and D. A. Winter, "Electromyographic amplitude normalization methods: improving their sensitivity as diagnostic tools in gait analysis," Archives of Physical Medicine and Rehabilitation, vol. 65, pp. 517-21, Sep. 1984.

[36] L. Bolgla and T. Uhl, "Reliability of electromyographic normalization methods for evaluating the hip musculature," Journal of Electromyography and Kinesiology, vol. 17, pp. 102-11, March 2007.

[37] A. Burden, M. Trew, and V. Baltzopoulos, "Normalisation of gait emgs: A re-examination," Journal of Electromyography and Kinesiology, vol. 13, pp. 519-32, Dec. 2003.

[38] A. Burden and R. Bartlett, "Normalisation of emg amplitude: an evaluation and comparison of old and new methods," Medical Engineering \& Physics, vol. 21, no. 4, pp. 247 - 257, May 1999.

[39] H. Graichen, K.-H. Englmeier, M. Reiser, and F. Eckstein, "An in vivo technique for determining $3 \mathrm{~d}$ muscular moment arms in different joint positions and during muscular activation - application to the supraspinatus," Clinical Biomechanics, vol. 16, no. 5, pp. 389 - 394, Jun. 2001.

[40] W. M. Murray, S. L. Delp, and T. S. Buchanan, "Variation of muscle moment arms with elbow and forearm position," Journal of Biomechanics, vol. 28, no. 5, pp. 513 - 525, May 1995

[41] Y. M. Aung and A. Al-Jumaily, "sEMG based ann for shoulder angle prediction," Procedia Engineering, vol. 41, pp. 1009 - 1015, Dec. 2012. 